PROCEEDINGS OF THE

AMERICAN MATHEMATICAL SOCIETY

Volume 125, Number 2, February 1997, Pages 463-470

S 0002-9939(97)03569-7

\title{
CONVOLUTION OF A MEASURE WITH ITSELF AND A RESTRICTION THEOREM
}

\author{
JONG-GUK BAK AND DAVID MCMICHAEL
}

(Communicated by J. Marshall Ash)

\begin{abstract}
Let $S_{k}=\left\{\left(y,|y|^{k}\right): y \in \mathbf{R}^{n-1}\right\} \subset \mathbf{R}^{n}$ and $\sigma$ be the measure defined by $\langle\sigma, \phi\rangle=\int_{\mathbf{R}^{n-1}} \phi\left(y,|y|^{k}\right) d y$. Let $\sigma_{P}$ denote the measure obtained by restricting $\sigma$ to the set $P=[0, \infty)^{n-1}$. We prove estimates on $\sigma_{P} * \sigma_{P}$. As a corollary we obtain results on the restriction to $S_{k} \subset \mathbf{R}^{3}$ of the Fourier transform of functions on $\mathbf{R}^{3}$ for $k \in \mathbf{R}, 2<k<6$.
\end{abstract}

\section{$\S 1$. INTRODUCTION}

Given a submanifold $S$ of $\mathbf{R}^{n}$ and a smooth measure $\sigma$ on $S$, one may ask for what values of $p$ and $q$ the $a$ priori estimate

$$
\left\|\left.\widehat{f}\right|_{S}\right\|_{L^{q}(\sigma)} \leq C_{p, q}\|f\|_{L^{p}\left(\mathbf{R}^{n}\right)}, \quad f \in \mathcal{S}\left(\mathbf{R}^{n}\right),
$$

holds. Here $\left.\widehat{f}\right|_{S}$ denotes the restriction of the Fourier transform of $f$ to $S$, and $\mathcal{S}\left(\mathbf{R}^{n}\right)$ is the Schwartz class of smooth, rapidly decreasing functions. Estimates of this type are known as restriction theorems. We refer to $[\mathrm{S}]$ and the references contained therein for the history and general discussion of these estimates. When $S$ is the unit circle in $\mathbf{R}^{2}(1.1)$ holds if and only if $1 \leq p<4 / 3$ and $1 / q \geq 3(1-1 / p)$ (Fefferman [F], Sjölin (see [H]), Zygmund [Z]). Fefferman's original proof of this result was based on a careful analysis of the convolution $g d \sigma * g d \sigma$, where $g$ is a function defined on the circle.

On the other hand restriction theorems for the unit sphere and other nondegenerate hypersurfaces in $\mathbf{R}^{n}$ have been obtained by an entirely different approach based on the observation by Tomas $[\mathrm{T}]$ that when $q=2$ the estimate (1.1) is equivalent to the estimate

$$
\|\widehat{\sigma} * f\|_{L^{p^{\prime}}\left(\mathbf{R}^{n}\right)} \leq C\|f\|_{L^{p}\left(\mathbf{R}^{n}\right)},
$$

where $p^{\prime}=p /(p-1)$ is the exponent conjugate to $p$. One can prove (1.2) for $p=p_{0}=2(n+1) /(n+3)$ by analytic interpolation (see $\left.[\mathrm{S}, \mathrm{Sz}]\right)$. This value of $p$ is sharp when $q=2$. (Interpolating this result with the trivial $L^{1}-L^{\infty}$ estimate gives the optimal value of $p$ when $q>2$.) But this approach fails to give new information for $q<2$. The important problem of obtaining sharp $L^{p}-L^{q}$ restriction estimates for the sphere in $\mathbf{R}^{n}(n \geq 3)$ is still open, although Bourgain [Bo] has recently made

Received by the editors April 13, 1995 and, in revised form, August 10, 1995.

1991 Mathematics Subject Classification. Primary 42B10.

The first author was supported in part by a grant from TGRC-KOSEF of Korea.

(C) 1997 American Mathematical Society 
major progress toward the solution of this problem by showing that there exists some $p>p_{0}$ such that (1.1) holds for $q=1$.

Consider the hypersurface $S_{k}=\left\{\left(y,|y|^{k}\right): y \in \mathbf{R}^{n-1}\right\}$ in $\mathbf{R}^{n}$ and the measure $d \mu=\chi d \sigma$, where $\langle\sigma, \phi\rangle=\int_{\mathbf{R}^{n-1}} \phi\left(y,|y|^{k}\right) d y$, and $\chi$ is a smooth cut-off function. When $q \leq 2$ and $k>2$, it is possible to use the result for $q=2$ in the nondegenerate case and a scaling argument to prove restriction estimates for $S_{k}$ when $p<p_{0}=$ $2(n+1) /(n+3)$. (This fact was pointed out to us by a referee to an earlier version of this paper. See $[B]$.

In this paper we prove (global) restriction estimates for $S_{k}$ when $n=3$ :

$$
\left\|\widehat{f}_{S_{k}}\right\|_{L^{8 /(k+2), \infty}(\sigma)} \leq C\|f\|_{L^{4 / 3}\left(\mathbf{R}^{3}\right)}
$$

if $k \in \mathbf{R}, 2<k<6$. (Here $L^{p, \infty}$ is the weak $L^{p}$ space.) Since $p_{0}=4 / 3$ when $n=3$, this is stronger than the result that can be obtained by the scaling argument mentioned above. This weak type estimate implies sharp Lorentz space estimates for the restriction of $\widehat{f}$ to $S_{k}$ when $f \in L^{p}\left(\mathbf{R}^{3}\right), p<4 / 3$. Our method of proof is similar in spirit to Fefferman's original proof for the circle: after an application of the Plancherel theorem we are led to estimate the convolution $\sigma_{P} * \sigma_{P}$, where $\sigma_{P}$ is the measure defined by $\left\langle\sigma_{P}, \phi\right\rangle=\int_{P} \phi\left(y,|y|^{k}\right) d y$ with $P=[0, \infty) \times[0, \infty)$. We show in section 2 how these estimates on $\sigma_{P} * \sigma_{P}$ imply (1.3), and prove the estimates on the convolution in section 3 .

A few words about notation. We let $C$ denote a positive constant whose actual value may vary. We use the symbol $a \lesssim b$ to indicate that $a \leq C b$. We write $a \approx b$ if $a \lesssim b$ and $b \lesssim a . L^{p, q}$ denotes a Lorentz space (see [SW]), and $|E|$ is the Lebesgue measure of the set $E$.

\section{$\S 2$. Statement of Results}

Consider the surface $S \subset \mathbf{R}^{3}$ given by $S=\left\{(y, \gamma(|y|)): y \in \mathbf{R}^{2}\right\}$, where $\gamma$ satisfies the following hypotheses.

(2.1) Hypotheses on $\gamma$. We assume $\gamma:[0, \infty) \rightarrow[0, \infty)$ is a $C^{2}$ function such that $\gamma(0)=\gamma^{\prime}(0)=0$ and $\gamma^{\prime}(r) / r$ is increasing for $r>0$, and that there exist a real number $k \geq 2$ and constants $0<C_{1} \leq C_{2}<\infty$ such that $C_{1} r^{k-2} \leq \gamma^{\prime \prime}(r) \leq C_{2} r^{k-2}$ for $r \geq 0$.

(2.2) Remark. It follows from (2.1) that $\gamma^{\prime}(r) \approx r^{k-1}, \gamma(r) \approx r^{k}$, and that $\gamma$ is strictly convex and strictly increasing. Also note that the condition that $\gamma^{\prime}(r) / r$ is increasing would follow if $\gamma^{\prime \prime \prime}(r) \geq 0$. (To see this note that if $\gamma^{\prime \prime \prime}(r) \geq 0$, then $\gamma^{\prime}(r)=\int_{0}^{r} \gamma^{\prime \prime}(s) d s \leq r \gamma^{\prime \prime}(r)$, and so $\gamma^{\prime}(r) / r$ has a positive derivative.)

We now state our restriction results.

(2.3) Theorem. Let $\sigma$ be the measure on $\mathbf{R}^{3}$ given by $\langle\sigma, \phi\rangle=\int_{\mathbf{R}^{2}} \phi(s, \gamma(|s|)) d s$, where $\gamma$ satisfies (2.1) for some real number $k \in(2,6)$. Then for all $f \in \mathcal{S}\left(\mathbf{R}^{3}\right)$

(a) $\left\|\left.\widehat{f}\right|_{S}\right\|_{L^{8 /(k+2), \infty(\sigma)}} \leq C\|f\|_{L^{4 / 3}\left(\mathbf{R}^{3}\right)}$;

(b) $\left\|\left.\widehat{f}\right|_{S}\right\|_{L^{q, p}(\sigma)} \leq C\|f\|_{L^{p}\left(\mathbf{R}^{3}\right)}$ for $p \in(1,4 / 3)$ and $q=2 p^{\prime} /(k+2)$.

Moreover, (b) is sharp in the sense that it fails if the $L^{q, p}$ norm on the left side is replaced by an $L^{q, s}$ norm for any $s<p$.

A simple homogeneity argument shows the relation $q=2 p^{\prime} /(k+2)$ is necessary in Theorem 2.3 (see e.g. [T, Sz]). (For $\delta>0$ take

$$
f_{\delta}(x)=\delta e^{-\pi\left(\delta x_{1}\right)^{2}} \delta e^{-\pi\left(\delta x_{2}\right)^{2}} \gamma(\delta) e^{-\pi\left(\gamma(\delta) x_{3}\right)^{2}} .
$$


Then $\widehat{f}_{\delta}(\xi)=e^{-\pi\left(\xi_{1} / \delta\right)^{2}} e^{-\pi\left(\xi_{2} / \delta\right)^{2}} e^{-\pi\left(\xi_{3} / \gamma(\delta)\right)^{2}}$, which is essentially the characteristic function of a box of dimensions $\delta \times \delta \times \gamma(\delta)$. Hence $\left\|\widehat{f}_{\delta}\right\|_{L^{q, \infty}(\sigma)} \approx \delta^{2 / q}$ and $\left\|f_{\delta}\right\|_{L^{p}\left(\mathbf{R}^{3}\right)} \approx \delta^{(k+2) /(1-1 / p)}$. So if $\left\|\left.\widehat{f}\right|_{S}\right\|_{L^{q, \infty}(\sigma)} \leq C\|f\|_{L^{p}\left(\mathbf{R}^{3}\right)}$ holds for $f \in \mathcal{S}\left(\mathbf{R}^{3}\right)$, then $\delta^{2 / q} \leq C \delta^{(k+2)(1-1 / p)}$ for all $\delta>0$. Therefore it follows that $q=2 p^{\prime} /(k+2)$. If the measure $\sigma$ is replaced by a compactly supported measure, then it is only necessary that $q \leq 2 p^{\prime} /(k+2)$.)

Let $P=[0, \infty) \times[0, \infty)$ and assume $\gamma$ satisfies (2.1). Define a function $G: P \times$ $P \rightarrow \mathbf{R}^{3}$ by

$$
G(s, t)=(s+t, \gamma(|s|)+\gamma(|t|))
$$

Then the measure $g d \sigma_{P}$ defined by $\left\langle g d \sigma_{P}, \phi\right\rangle=\int_{P} \phi(s, \gamma(|s|)) g(s) d s$ satisfies (see [GS, p. 103])

$$
\begin{aligned}
\left\langle g d \sigma_{P} * g d \sigma_{P}, \phi\right\rangle & =\int_{P} \int_{P} \phi((t, \gamma(|t|))+(s, \gamma(|s|))) g(s) d s g(t) d t \\
& =\int_{P \times P} \phi(G(s, t)) g(s) g(t) d s d t .
\end{aligned}
$$

In Lemma 2.6 below we prove estimates on the mapping $\phi \mapsto \phi \circ G$, which therefore imply estimates on $g d \sigma_{P} * g d \sigma_{P}$. We first state this lemma, and then show how it can be used to prove Theorem 2.3. The proof of Lemma 2.6 is then given in the third section of the paper.

(2.6) Lemma. Let $G$ be defined by (2.4), where $\gamma$ satisfies (2.1) for some $k \in$ $[2, \infty)$. Then

(a) $\|\phi \circ G\|_{L^{1}(P \times P)} \leq C\|\phi\|_{L^{(k+2) / 4,1}\left(\mathbf{R}^{3}\right)}$;

(b) $\|\phi \circ G\|_{L^{q,(k+2) q / 4}(P \times P)} \leq C\|\phi\|_{L^{(k+2) q / 4}\left(\mathbf{R}^{3}\right)}$ if $1<q<\infty$.

Proof of Theorem 2.3. We first note that 2.3(b) follows from 2.3(a) and the trivial $L^{1}-L^{\infty}$ estimate by interpolation.

Also a straightforward generalization of calculations given by Sogge [So], applied to the functions $g_{\delta}(s)=\chi_{[0, \delta]}(|s|)|s|^{-2 / p}(\log 1 /|s|)^{1 / q}$, for small $\delta>0$, implies the sharpness results. We omit the details.

To prove 2.3(a) consider an operator $T$ given by

$$
T g(\xi)=\int_{\mathbf{R}^{2}} e^{2 \pi i \xi \cdot(s, \gamma(|s|))} g(s) d s=(g d \sigma)^{\wedge}(\xi),
$$

where $g \in L^{1}\left(\mathbf{R}^{2}\right)$ and $\xi \in \mathbf{R}^{3}$. By duality 2.3(a) follows from the following estimate for $T$ :

$$
\|T g\|_{L^{4}\left(\mathbf{R}^{3}\right)} \leq C\|g\|_{L^{8 /(6-k), 1}\left(\mathbf{R}^{2}\right)}
$$

Let $\sigma_{P}$ be defined by $\left\langle\sigma_{P}, \phi\right\rangle=\int_{P} \phi(s, \gamma(|s|)) d s$, where $P=[0, \infty) \times[0, \infty)$ as above. Observe that it suffices to prove (2.7) with $T$ replaced by $\widetilde{T}$, where

$$
\widetilde{T} g(\xi)=\int_{P} e^{2 \pi i \xi \cdot(s, \gamma(|s|))} g(s) d s=\left(g d \sigma_{P}\right)^{\wedge}(\xi) .
$$

In order to prove the estimate for $\widetilde{T}$ we may assume $g$ is the characteristic function of a Borel set $E$ (see [SW, p. 195]). We have

$$
\begin{aligned}
\left\|\widetilde{T} \chi_{E}\right\|_{L^{4}\left(\mathbf{R}^{3}\right)}^{2}=\left\|\left(\widetilde{T} \chi_{E}\right)^{2}\right\|_{L^{2}\left(\mathbf{R}^{3}\right)} & =\left\|\left(\chi_{E} d \sigma_{P} * \chi_{E} d \sigma_{P}\right)^{\wedge}\right\|_{L^{2}\left(\mathbf{R}^{3}\right)} \\
& =\left\|\chi_{E} d \sigma_{P} * \chi_{E} d \sigma_{P}\right\|_{L^{2}\left(\mathbf{R}^{3}\right)}
\end{aligned}
$$


by the Plancherel theorem. To estimate the last $L^{2}$ norm we let it act on test functions $\phi$. Hölder's inequality for Lorentz spaces (see $[\mathrm{O}]$ ) and Lemma 2.6(b) with $q=8 /(k+2)$ give

$$
\begin{aligned}
\left|\left\langle\chi_{E} d \sigma_{P} * \chi_{E} d \sigma_{P}, \phi\right\rangle\right| & =\left|\int_{P \times P} \phi(G(s, t)) \chi_{E}(s) \chi_{E}(t) d s d t\right| \\
& \leq C\|\phi \circ G\|_{L^{8 /(k+2), \infty}(P \times P)}\left\|\chi_{E}(s) \chi_{E}(t)\right\|_{L^{8 /(6-k), 1}(P \times P)} \\
& \leq C\|\phi \circ G\|_{L^{8 /(k+2), 2(P \times P)}}|E|^{2(6-k) / 8} \\
& \leq C\|\phi\|_{L^{2}\left(\mathbf{R}^{3}\right)}\left\|\chi_{E}\right\|_{L^{8 /(6-k)}(P)}^{2} .
\end{aligned}
$$

Thus $\left\|\chi_{E} d \sigma_{P} * \chi_{E} d \sigma_{P}\right\|_{L^{2}\left(\mathbf{R}^{3}\right)} \leq C\left\|\chi_{E}\right\|_{L^{8 /(6-k)}(P)}^{2}$, and hence $\left\|\widetilde{T} \chi_{E}\right\|_{L^{4}\left(\mathbf{R}^{3}\right)} \leq$ $C\left\|\chi_{E}\right\|_{L^{8 /(6-k)(P)}}$, which finishes the proof of (2.7).

\section{§3. Proof of Lemma 2.6}

We will deduce Lemma 2.6 from part (d) of the next lemma, which follows from certain pointwise estimates (see (3.11) below) for the convolution $\sigma_{P} * \sigma_{P}$.

Notation. Write $x=\left(x^{\prime}, z\right)$ with $x^{\prime}=\left(x_{1}, x_{2}\right)$ and $z=x_{3}$. For $\xi, y \in \mathbf{R}^{2}$ let $F_{\xi}(y)=\gamma(|\xi+y| / 2)+\gamma(|\xi-y| / 2)$.

(3.1) Lemma. Assume that $\gamma$ satisfies (2.1) and let $P, G$ and $\sigma_{P}$ be as above. Then

(a) $G(P \times P)=\left\{x \in \mathbf{R}^{3}: x^{\prime} \in P, 2 \gamma\left(\left|x^{\prime}\right| / 2\right) \leq z \leq \gamma\left(\left|x^{\prime}\right|\right)\right\}$;

(b) $\sigma_{P} * \sigma_{P}$ is absolutely continuous with respect to Lebesgue measure in $\mathbf{R}^{3}$;

(c) $\sigma_{P} * \sigma_{P}(x)=0$ if $x \notin G(P \times P)$, and

$$
\sigma_{P} * \sigma_{P}(x)=\frac{1}{4} \int_{R_{x^{\prime}} \cap F_{x^{\prime}}^{-1}(z)}\left|\nabla F_{x^{\prime}}(y)\right|^{-1} d S(y)
$$

if $x$ lies in the interior of $G(P \times P)$;

(d) $\sigma_{P} * \sigma_{P} \in L^{(k+2) /(k-2), \infty}\left(\mathbf{R}^{3}\right)$ if $k>2$;

(e) $\sigma_{P} * \sigma_{P} \in L^{\infty}\left(\mathbf{R}^{3}\right)$ if $k=2$.

In (c) above $d S$ denotes the arc length measure on the level curve $F_{x^{\prime}}^{-1}(z)=$ $\left\{y: F_{x^{\prime}}(y)=z\right\}$ and the gradient is taken with respect to $y$. Let us assume Lemma 3.1 for the moment and prove Lemma 2.6.

Proof of Lemma 2.6. To prove 2.6(a) we may take $\phi$ to be a characteristic function of a set $E \subset \mathbf{R}^{3}$ (see [SW, p. 195]). From (2.5) it follows that

$$
\left\|\chi_{E} \circ G\right\|_{L^{1}(P \times P)}=\int_{P \times P} \chi_{E}(G(s, t)) d s d t=\left\langle\sigma_{P} * \sigma_{P}, \chi_{E}\right\rangle .
$$

Hence, if $k>2$, Hölder's inequality and Lemma 3.1 give

$$
\begin{aligned}
\left\|\chi_{E} \circ G\right\|_{L^{1}(P \times P)}=\left\langle\sigma_{P} * \sigma_{P}, \chi_{E}\right\rangle & \leq C\left\|\sigma_{P} * \sigma_{P}\right\|_{L^{(k+2) /(k-2), \infty}}\left\|\chi_{E}\right\|_{L^{(k+2) / 4,1}} \\
& \leq C\left\|\chi_{E}\right\|_{L^{(k+2) / 4,1}} .
\end{aligned}
$$

This finishes the proof of 2.6(a).

Now applying the Marcinkiewicz interpolation theorem for Lorentz spaces to 2.6(a) and the estimate $\|\phi \circ G\|_{L^{\infty}(P \times P)} \leq\|\phi\|_{L^{\infty}\left(\mathbf{R}^{n}\right)}$ gives 2.6(b). 
Proof of Lemma 3.1. We begin with a few observations concerning the geometry of the level curves of the function $F_{\xi}$. Fix a nonzero vector $\xi=\left(\xi_{1}, \xi_{2}\right)$ and let $\eta=\left(-\xi_{2}, \xi_{1}\right)$. First we note that (2.1) implies that $F_{\xi}$ is a convex function. Hence the level curves of $F_{\xi}$ bound convex domains. Next observe that these curves are symmetric about the line $L_{\xi}$ which passes through the origin and $\xi$, and also about the line $L_{\eta}$ passing through the origin and $\eta$. (If $y^{\prime}$ is the reflection of $y$ through $L_{\xi}$, then $\left|y^{\prime}+\xi\right|=|y+\xi|$ and $\left|y^{\prime}-\xi\right|=|y-\xi|$, while if $y^{\prime \prime}$ is the reflection of $y$ through $L_{\eta}$, then $\left|y^{\prime \prime}+\xi\right|=|y-\xi|$ and $\left|y^{\prime \prime}-\xi\right|=|y+\xi|$.) The third observation is that these level curves are "elliptical" in shape.

To be more precise, we claim that

(i) For each vector $\nu \neq 0$, the function $r \mapsto F_{\xi}(r \nu)$ is a strictly increasing function from $[0, \infty)$ onto $[2 \gamma(|\xi| / 2), \infty)$.

(ii) Fix $z>2 \gamma(|\xi| / 2)=F_{\xi}(0)$. Let $y_{-}$be the point on the level curve $F_{\xi}^{-1}(z)$ which lies on the ray $\{r \xi: r \geq 0\}$, and $y_{+}$the point on this level curve which lies on the ray $\{r \eta: r \geq 0\}$. Then if $y$ is any point on this level curve, we have $\left|y_{-}\right| \leq|y| \leq\left|y_{+}\right|$.

To see (i) note that

$$
\nabla F_{\xi}(y)=(\alpha-\beta) \xi+(\alpha+\beta) y,
$$

where $\alpha=\gamma^{\prime}(|\xi+y| / 2) /(2|\xi+y|)$ and $\beta=\gamma^{\prime}(|\xi-y| / 2) /(2|\xi-y|)$. Also, (2.1) implies that $(\alpha-\beta) x^{\prime} \cdot y \geq 0$, since $\xi \cdot y \geq 0$ if and only if $|\xi+y| \geq|\xi-y|$. Therefore $\nabla F_{\xi}(y) \cdot y=(\alpha-\beta) \xi \cdot y+(\alpha+\beta)|y|^{2}>0$ if $y \neq 0$.

Part (ii) follows easily from the following inequality. For $y \in \mathbf{R}^{2}$ define $\tilde{y}=$ $|y| \eta /|\eta|$ and $y^{*}=|y| \xi /|\xi|$. Then

$$
F_{\xi}(\tilde{y}) \leq F_{\xi}(y) \leq F_{\xi}\left(y^{*}\right) .
$$

To prove (3.4), define a function $\psi(r)=\gamma(\sqrt{r} / 2)$ for $r \geq 0$. Since $\gamma^{\prime}(r) / r$ is increasing by (2.1), $\psi$ has an increasing derivative, and hence is convex. Therefore

$$
\begin{aligned}
2 \psi\left(|\xi|^{2}+|y|^{2}\right) & \leq \psi\left(|\xi|^{2}+|y|^{2}-2 \xi \cdot y\right)+\psi\left(|\xi|^{2}+|y|^{2}+2 \xi \cdot y\right) \\
& \leq \psi\left(|\xi|^{2}+|y|^{2}-2 \xi \cdot y^{*}\right)+\psi\left(|\xi|^{2}+|y|^{2}+2 \xi \cdot y^{*}\right),
\end{aligned}
$$

which is equivalent to (3.4).

To prove 3.1(a), first suppose that $x=\left(x^{\prime}, z\right)=\left(x_{1}, x_{2}, z\right) \in G(P \times P)$. Then there exist points $s, t \in P$ such that $G(s, t)=(t+s, \gamma(|t|)+\gamma(|s|))=\left(x^{\prime}, z\right)$, and so $x^{\prime}=t+s$ and $z=\gamma(|t|)+\gamma(|s|)$. If we put $v=t-s$ for these $s, t$, then $z=F(v)$, where $F \equiv F_{x^{\prime}}$ and $v=\left(v_{1}, v_{2}\right)$ satisfies $\left|v_{j}\right| \leq x_{j}$ for $j=1,2$. Since $x^{\prime} \in P$, to show that $G(P \times P)$ is a subset of the set on the right side of (a), it suffices to prove

$$
2 \gamma\left(\left|x^{\prime}\right| / 2\right) \leq F(v) \leq \gamma\left(\left|x^{\prime}\right|\right)
$$

for $x^{\prime} \neq 0$. However these inequalities follow easily from (i) above and (3.4), since $2 \gamma\left(\left|x^{\prime}\right| / 2\right)=F(0) \leq F(v) \leq F\left(|v| x^{\prime} /\left|x^{\prime}\right|\right) \leq F\left(\left|x^{\prime}\right| x^{\prime} /\left|x^{\prime}\right|\right)=F\left(x^{\prime}\right)=\gamma\left(\left|x^{\prime}\right|\right)$.

Conversely, if $x^{\prime} \in P$ and $2 \gamma\left(\left|x^{\prime}\right| / 2\right) \leq z \leq \gamma\left(\left|x^{\prime}\right|\right)$, then there exists $b \in[0,1]$ such that $F\left(b x^{\prime}\right)=z$, since $F(0)=2 \gamma\left(\left|x^{\prime}\right| / 2\right)$ and $F\left(x^{\prime}\right)=\gamma\left(\left|x^{\prime}\right|\right)$ and $F$ is continuous. Now $\left.G\left((1-b) x^{\prime} / 2\right),(1+b) x^{\prime} / 2\right)=\left(x^{\prime}, F\left(b x^{\prime}\right)\right)=\left(x^{\prime}, z\right)$. This shows $\left(x^{\prime}, z\right) \in$ $G(P \times P)$ and finishes the proof of (a).

Next we prove 3.1(b). We need to show that $\left\langle\sigma_{P} * \sigma_{P}, \chi_{E}\right\rangle=0$ for each set $E$ of Lebesgue measure zero in $\mathbf{R}^{3}$. The change of variables $\xi_{j}=t_{j}+s_{j}, y_{j}=t_{j}-s_{j}$ 
$(j=1,2)$ gives

$$
\begin{aligned}
\left\langle\sigma_{P} * \sigma_{P}, \chi_{E}\right\rangle & =\int_{P \times P} \chi_{E}(G(s, t)) d s d t \\
& =\frac{1}{4} \int_{-\infty}^{\infty} \int_{\left|y_{2}\right|}^{\infty} \int_{0}^{\infty} \int_{-\xi_{1}}^{\xi_{1}} \chi_{E}\left(\xi_{1}, \xi_{2}, F_{\xi}\left(y_{1}, y_{2}\right)\right) d y_{1} d \xi_{1} d \xi_{2} d y_{2} .
\end{aligned}
$$

For each fixed $y_{2}$ and $\xi, F_{\xi}\left(y_{1}, y_{2}\right)$ is a strictly convex function of $y_{1}$. Hence the change of variables $y_{1} \mapsto u$ given by $u=F_{\xi}\left(y_{1}, y_{2}\right)$ shows that the triple integral in $\left(y_{1}, \xi_{1}, \xi_{2}\right)$ is zero (for each $\left.y_{2}\right)$, since $|E|=0$.

The first assertion in 3.1(c) is obvious because of (a) and (2.5). To prove (3.2) note that, in view of the absolute continuity of $\sigma_{P} * \sigma_{P}$ just established, it may be calculated by the relation

$$
\sigma_{P} * \sigma_{P}(x)=\lim _{a \downarrow 0} \frac{1}{\left|Q_{a}\right|}\left\langle\sigma_{P} * \sigma_{P}, \chi_{Q_{a}}\right\rangle=\lim _{a \downarrow 0} \frac{1}{\left|Q_{a}\right|} \int_{P \times P} \chi_{Q_{a}}(G(s, t)) d s d t \quad \text { a.e. }
$$

where $Q_{a}=Q_{a}(x)=\left[x_{1}, x_{1}+a\right] \times\left[x_{2}, x_{2}+a\right] \times[z, z+a]$.

By the change of variables $\xi_{j}=t_{j}+s_{j}, y_{j}=t_{j}-s_{j}(j=1,2)$,

$$
\begin{aligned}
\int_{P \times P} \chi_{Q_{a}}(G(s, t)) d s d t & =\frac{1}{4} \int_{P} \int_{R_{\xi}} \chi_{Q_{a}}\left(\xi, F_{\xi}(y)\right) d y d \xi \\
& =\frac{1}{4} \int_{S_{x, a}} \int_{R_{\xi} \cap\left\{y: z \leq F_{\xi}(y) \leq z+a\right\}} d y d \xi,
\end{aligned}
$$

where $R_{\xi}=\left\{y \in \mathbf{R}^{2}:\left|y_{j}\right| \leq \xi_{j}, j=1,2\right\}$ and $S_{x, a}=\left\{\xi \in \mathbf{R}^{2}: x_{j} \leq \xi_{j} \leq\right.$ $\left.x_{j}+a, j=1,2\right\}$. By applying the coarea formula (see [Fe, p. 249]) to the $y$ integral, for example, and then the mean value theorem for integrals, we see that

$$
\begin{aligned}
\frac{1}{\left|Q_{a}\right|} \int_{P \times P} \chi_{Q_{a}}(G(s, t)) d s d t & =\frac{1}{4 a^{3}} \int_{S_{x, a}} \int_{z}^{z+a} \int_{R_{\xi} \cap F_{\xi}^{-1}(u)} \frac{d S(y)}{\left|\nabla F_{\xi}(y)\right|} d u d \xi \\
& =\frac{1}{4} \int_{R_{\xi_{0}} \cap F_{\xi_{0}}^{-1}\left(u_{0}\right)} \frac{d S(y)}{\left|\nabla F_{\xi_{0}}(y)\right|}
\end{aligned}
$$

for some $\xi_{0} \in S_{x, a}$ and $u_{0} \in[z, z+a]$. Here $d S=d S_{\xi, u}$ denotes arc length measure on the level curve $F_{\xi}^{-1}(u)$, and $\nabla$ is the gradient with respect to $y$. Hence taking the limit as $a \downarrow 0$ gives (3.2). Thus we have

$$
\sigma_{P} * \sigma_{P}(x) \leq \frac{1}{4} \int_{F^{-1}(z)} d S \cdot \max \left\{|\nabla F(y)|^{-1}: F(y)=z\right\} .
$$

By (a), (b) and symmetry, to prove (d) and (e) it is enough to estimate $\sigma_{P} * \sigma_{P}(x)$ at points $x=\left(x^{\prime}, z\right)$, where $2 \gamma\left(\left|x^{\prime}\right| / 2\right)<z<\gamma\left(\left|x^{\prime}\right|\right)$ and $0<x_{1} \leq x_{2}$.

We will first prove an estimate for $\int_{F^{-1}(z)} d S$. If $2 \gamma\left(\left|x^{\prime}\right| / 2\right)<z<\gamma\left(\left|x^{\prime}\right|\right)$, then (i), (ii), (3.4) (with $\left.\xi=x^{\prime}\right)$ and the facts that $F(0)=2 \gamma\left(\left|x^{\prime}\right| / 2\right), F\left(x^{\prime}\right)=\gamma\left(\left|x^{\prime}\right|\right)$ imply that there exists a unique $\lambda \in(0,1)$ such that $y_{-}=\lambda x^{\prime}$ satisfies $F\left(y_{-}\right)=z$ and $\left|y_{-}\right|=\min \{|y|: F(y)=z\}$. It also follows from (ii) that the maximum value of $|y|$ on the curve $F(y)=z$ occurs at a point $y_{+}$which satisfies $x^{\prime} \cdot y_{+}=0$. From the relation $z=F\left(y_{+}\right)=2 \gamma\left(\sqrt{\left|x^{\prime}\right|^{2}+\left|y_{+}\right|^{2}} / 2\right)$ we get $\left|y_{+}\right|^{2}=4\left[\gamma^{-1}(z / 2)\right]^{2}-\left|x^{\prime}\right|^{2}$. Since $F^{-1}(z)$ is a curve which bounds a convex domain and $F^{-1}(z)$ is contained in 
the annulus $\left\{y \in \mathbf{R}^{2}:\left|y_{-}\right| \leq|y| \leq\left|y_{+}\right|\right\}$, we conclude that

$$
\int_{F^{-1}(z)} d S \leq 2 \pi\left|y_{+}\right| \text {. }
$$

Since $2 \gamma\left(\left|x^{\prime}\right| / 2\right)<z<\gamma\left(\left|x^{\prime}\right|\right),(2.2)$ implies that $z \approx\left|x^{\prime}\right|^{k}$ and $\gamma^{-1}(z / 2) \approx\left|x^{\prime}\right|$. By the mean value theorem

$$
\begin{aligned}
\left|y_{+}\right|^{2} & =4\left[\gamma^{-1}(z / 2)+\left|x^{\prime}\right| / 2\right]\left[\gamma^{-1}(z / 2)-\gamma^{-1}\left(\gamma\left(\left|x^{\prime}\right| / 2\right)\right)\right] \\
& =4\left[\gamma^{-1}(z / 2)+\left|x^{\prime}\right| / 2\right]\left[z / 2-\gamma\left(\left|x^{\prime}\right| / 2\right)\right] / \gamma^{\prime}\left(\gamma^{-1}(\zeta)\right)
\end{aligned}
$$

for some $\zeta$ between $\gamma\left(\left|x^{\prime}\right| / 2\right)$ and $z / 2$. Since $\zeta \approx\left|x^{\prime}\right|^{k}$, we have $\gamma^{\prime}\left(\gamma^{-1}(\zeta)\right) \approx\left|x^{\prime}\right|^{k-1}$ by $(2.2)$, and so

$$
\left|y_{+}\right| \approx\left|x^{\prime}\right|^{1-k / 2}\left[z-2 \gamma\left(\left|x^{\prime}\right| / 2\right)\right]^{1 / 2} .
$$

Hence

$$
\int_{F^{-1}(z)} d S \leq C\left|x^{\prime}\right|^{1-k / 2}\left[z-2 \gamma\left(\left|x^{\prime}\right| / 2\right)\right]^{1 / 2} .
$$

Next we will prove

$$
\max \left\{|\nabla F(y)|^{-1}: F(y)=z\right\} \leq C\left|x^{\prime}\right|^{1-k / 2}\left[z-2 \gamma\left(\left|x^{\prime}\right| / 2\right)\right]^{-1 / 2} .
$$

From (3.3) we have

$$
|\nabla F(y)|^{2}=(\alpha-\beta)^{2}\left|x^{\prime}\right|^{2}+2(\alpha+\beta)(\alpha-\beta) x^{\prime} \cdot y+(\alpha+\beta)^{2}|y|^{2},
$$

and so we have $|\nabla F(y)| \geq(\alpha+\beta)|y|$, since $(\alpha-\beta) x^{\prime} \cdot y \geq 0$. By the above argument following (3.5), if $F(y)=z$ then

$$
|\nabla F(y)| \geq(\alpha+\beta) \min \{|y|: F(y)=z\}=(\alpha+\beta)\left|y_{-}\right|,
$$

where $y_{-}=\lambda x^{\prime}$ is as above. We will now obtain a lower bound for $\left|y_{-}\right|$. Observe that by the mean value theorem

$$
\begin{aligned}
z-2 \gamma\left(\left|x^{\prime}\right| / 2\right) & =F\left(y_{-}\right)-2 \gamma\left(\left|x^{\prime}\right| / 2\right)=F\left(\lambda x^{\prime}\right)-2 \gamma\left(\left|x^{\prime}\right| / 2\right) \\
& =\gamma\left((1+\lambda)\left|x^{\prime}\right| / 2\right)-\gamma\left(\left|x^{\prime}\right| / 2\right)+\gamma\left((1-\lambda)\left|x^{\prime}\right| / 2\right)-\gamma\left(\left|x^{\prime}\right| / 2\right) \\
& =\lambda\left|x^{\prime}\right| \gamma^{\prime}\left(w_{1}\right) / 2-\lambda\left|x^{\prime}\right| \gamma^{\prime}\left(w_{2}\right) / 2
\end{aligned}
$$

for some $w_{1}, w_{2}$ with $\left|x^{\prime}\right| / 2<w_{1}<(1+\lambda)\left|x^{\prime}\right| / 2$ and $(1-\lambda)\left|x^{\prime}\right| / 2<w_{2}<\left|x^{\prime}\right| / 2$. Therefore $z-2 \gamma\left(\left|x^{\prime}\right| / 2\right)=\left[\gamma^{\prime}\left(w_{1}\right)-\gamma^{\prime}\left(w_{2}\right)\right] \lambda\left|x^{\prime}\right| / 2=\left(w_{1}-w_{2}\right) \gamma^{\prime \prime}\left(w_{3}\right) \lambda\left|x^{\prime}\right| / 2$ for some $w_{3}$ with $w_{2}<w_{3}<w_{1}$. It then follows from (2.1) that $z-2 \gamma\left(\left|x^{\prime}\right| / 2\right) \lesssim$ $\lambda\left|x^{\prime}\right| \cdot\left|x^{\prime}\right|^{k-2} \cdot \lambda\left|x^{\prime}\right|=\lambda^{2}\left|x^{\prime}\right|^{k}$. Thus $\lambda \geq C\left|x^{\prime}\right|^{-k / 2}\left[z-2 \gamma\left(\left|x^{\prime}\right| / 2\right)\right]^{1 / 2}$, and hence

$$
\left|y_{-}\right|=\lambda\left|x^{\prime}\right| \geq C\left|x^{\prime}\right|^{1-k / 2}\left[z-2 \gamma\left(\left|x^{\prime}\right| / 2\right)\right]^{1 / 2} .
$$

(This together with the estimate for $\left|y_{+}\right|$shows that

$$
\left|y_{-}\right| \approx\left|y_{+}\right| \approx\left|x^{\prime}\right|^{1-k / 2}\left[z-2 \gamma\left(\left|x^{\prime}\right| / 2\right)\right]^{1 / 2} .
$$

That is, the eccentricity of the level curve $F_{x^{\prime}}^{-1}(z)$ stays bounded as the point $x=\left(x^{\prime}, z\right)$ varies.) Now $(2.2)$ implies that

$$
\begin{aligned}
& \alpha+\beta=\gamma^{\prime}\left(\left|x^{\prime}+y\right| / 2\right) /\left(2\left|x^{\prime}+y\right|\right)+\gamma^{\prime}\left(\left|x^{\prime}-y\right| / 2\right) /\left(2\left|x^{\prime}-y\right|\right) \\
& \quad \approx\left|x^{\prime}+y\right|^{k-2}+\left|x^{\prime}-y\right|^{k-2} \geq\left|x_{2}+y_{2}\right|^{k-2}+\left|x_{2}-y_{2}\right|^{k-2} \geq x_{2}^{k-2} \approx\left|x^{\prime}\right|^{k-2} .
\end{aligned}
$$

From this estimate, (3.9), and (3.10), we conclude that if $F(y)=z$, then $|\nabla F(y)| \geq$ $C\left|x^{\prime}\right|^{k / 2-1}\left[z-2 \gamma\left(\left|x^{\prime}\right| / 2\right)\right]^{1 / 2}$. This proves (3.8). 
Thus from (3.5), (3.7), and (3.8) we conclude that if $0<x_{1} \leq x_{2}$, then

$$
\sigma_{P} * \sigma_{P}(x) \leq C\left|x^{\prime}\right|^{2-k} \leq C x_{2}^{2-k} \quad \text { if } \quad 2 \gamma\left(\left|x^{\prime}\right| / 2\right)<z<\gamma\left(\left|x^{\prime}\right|\right) .
$$

From this estimate, 3.1(e) is obvious. When $k>2$ we have

$$
\begin{aligned}
\left|\left\{x \in \mathbf{R}^{3}: \sigma_{P} * \sigma_{P}(x)>\varepsilon\right\}\right| & \leq C \int_{0}^{C \varepsilon^{1 /(2-k)}} \int_{0}^{x_{2}} \int_{2 \gamma\left(\left|x^{\prime}\right| / 2\right)}^{\gamma\left(\left|x^{\prime}\right|\right)} d z d x_{1} d x_{2} \\
& \leq C \varepsilon^{-(k+2) /(k-2)}
\end{aligned}
$$

for $\varepsilon>0$. This yields 3.1(d).

Finally we would like to note that under the same hypotheses (2.1) for $\gamma$, an $n$-dimensional version $(n \geq 3)$ of Lemma 3.1 still holds with a similar proof. The estimate (3.11) should then be replaced by

$$
\sigma_{P} * \sigma_{P}(x) \leq C\left|x^{\prime}\right|^{n-1-k} \quad \text { if } \quad 2 \gamma\left(\left|x^{\prime}\right| / 2\right)<x_{n}<\gamma\left(\left|x^{\prime}\right|\right),
$$

where $x^{\prime}=\left(x_{1}, \ldots, x_{n-1}\right)$. The analog of Lemma 3.1(d) becomes

$$
\sigma_{P} * \sigma_{P} \in L^{(k+n-1) /(k-n+1), \infty}\left(\mathbf{R}^{n}\right) \quad \text { if } \quad k>n-1 .
$$

\section{REFERENCES}

[B] J.-G. Bak, Sharp convolution estimates for measures on flat surfaces, J. Math. Anal. Appl. 193 (1995), 756-771. CMP 95:15

[Bo] J. Bourgain, Besicovitch-type maximal operators and applications to Fourier analysis, Geom. and Funct. Anal. 1 (1991), 147-187. MR 92g:42010

[Fe] H. Federer, Geometric Measure Theory, Springer-Verlag, New York, 1969. MR 41:1976

[F] C. Fefferman, Inequalities for strongly singular convolution operators, Acta Math. 124 (1970), 9-36. MR 41:2468

[GS] I. M. Gelfand and G. E. Shilov, Generalized Functions, Vol. I, Academic Press, New York, 1964. MR 29:3869

[H] L. Hörmander, Oscillatory integrals and multipliers on $F L^{p}$, Ark. Mat. 11 (1973), 1-11. MR 49:5674

[O] R. O'Neil, Convolution operators and L(p,q) spaces, Duke Math. J. 30 (1963), 129-142. MR 26:4193

[So] C. Sogge, A sharp restriction theorem for degenerate curves in $\mathbf{R}^{2}$, Amer. J. Math. 109 (1987), 223-228. MR 88e:42027

[S] E. M. Stein, Harmonic Analysis: Real-Variable Methods, Orthogonality, and Oscillatory Integrals, Princeton Univ. Press, Princeton, NJ, 1993. MR 95c:42002

[SW] E. M. Stein and G. Weiss, Introduction to Fourier Analysis on Euclidean Spaces, Princeton Univ. Press, Princeton, NJ, 1971. MR 46:4102

[Sz] R. S. Strichartz, Restrictions of Fourier transforms to quadratic surfaces and decay of solutions of wave equations, Duke Math. J. 44 (1977), 705-713. MR 58:23577

[T] P. Tomas, Restriction theorems for the Fourier transform, in Proceedings of Symposia in Pure Mathematics, Vol. 35, pp. 111-114, Amer. Math. Soc., 1979. MR 81d:42029

[Z] A. Zygmund, On Fourier coefficients and transforms of two variables, Studia Math. 50 (1974), 189-201. MR 52:8788

Department of Mathematics, Florida State University, Tallahassee, Florida 32306 Current address, J.-G. Bak: Department of Mathematics, Pohang University of Science and Technology, Pohang 790-784, Korea

E-mail address, J.-G. Bak: bak@euclid.postech.ac.kr 